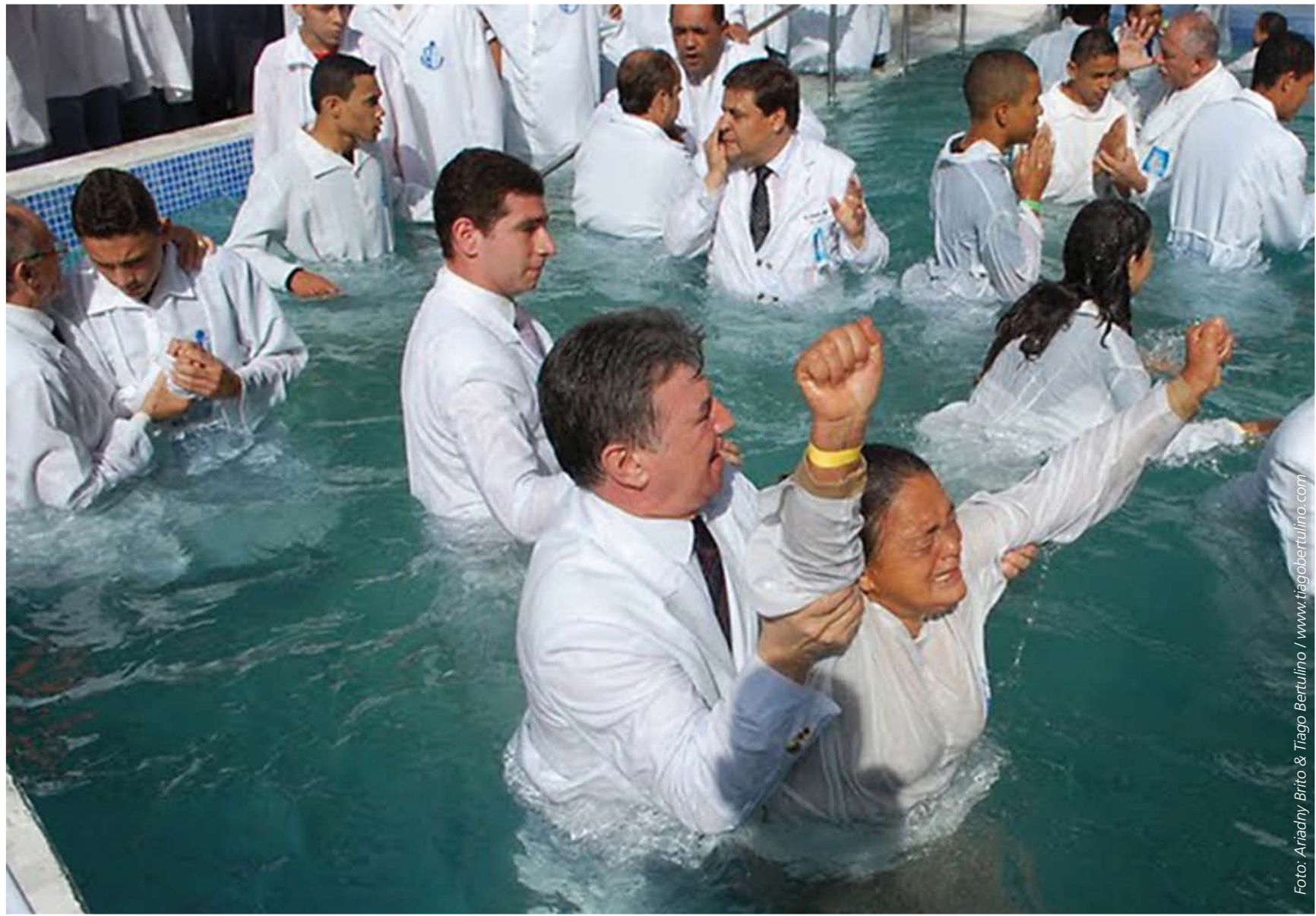

\title{
Macht und Ohnmacht des Heiligen Geistes
}

In Ländern wie Guatemala und Nicaragua folgt ein großer Teil der Bevölkerung der Pfingstbewegung. Begegnungen mit dieser Glaubensrichtung geben der Forschung Einblicke in ein Wechselspiel von Glaubenspraxis, sozialer Ungleichheit und Gewalt.

rüder, wir stehen vor der Realität der Agenten des Satans mit ihren außerordentlichen und übernatürlichen Mächten. (...) Und wir sind die Agenten Gottes, des Allmächtigen! Also, ich glaube, Brüder, wir stehen am Beginn einer weltweiten Auseinandersetzung zwischen den satanischen Mächten und ihren Agenten und der genuinen Macht der Agenten Gottes.“

Was in seiner Kriegsrhetorik einer Rundfunkansprache der Hisbollah entliehen sein könnte, hat ein pfingstkirchlicher Prediger aus der oberen Mittelschicht im Bürgerkrieg Guatemalas formuliert. Seine Aussage steht für die zunehmende Präsenz religiöser Bewegungen und Organisationen im politischen Leben weltweit. Sie setzt Lebensbedingungen voraus, in denen eine religiöse Deutung und Bewältigung der Lebenslage sinnvoll zu sein scheint.

Ganz anderes dagegen weiß ein verarmter indianischer Bauer zu 
berichten: „Meinen Schwager holten die Soldaten nachts aus seinem Haus. Am anderen Morgen wurde er gefoltert und mit durchgeschnittener Kehle gefunden. (...) Nun, die Bibel selbst sagt, wenn sich das Ende der Welt nähert, wird alles dieses geschehen."

Beide Äußerungen stammen aus der Zeit der Bürgerkriege in Guatemala und Nicaragua, aufgenommen in einem Forschungsprojekt des Verfassers Mitte der 1980er-Jahre. Sie deuten auf charakteristische Unterschiede hin, die mit der sozialen Stellung der Sprecher zusammenhängen.

Der erste, ein professioneller Prediger, gehört der oberen Mittelschicht an, die durch Krieg und Wirtschaftskrise um ihre Aufstiegsperspektiven gebracht wurde. Er schwört sich und seine Glaubensgeschwister auf eine Strategie der Macht und des Einflusses ein: Wir können alle die Mächte der Finsternis fesseln ...! Der zweite optiert für einen Rückzug in die Kirchengemeinde: Das ist die ganze Sicherheit, die einer hat: sich vorbereiten, um in der Begegnung mit dem Herrn dabei sein zu können. Beide Männer gehören der sogenannten Pfingstbewegung an, die sich seit den 1960er-Jahren in Lateinamerika stark verbreitet hat. Diese religiöse Bewegung setzt auf ekstatische Erfahrung des Heiligen Geistes, erwartet das Kommen des Reiches Gottes und betont häufig die Autonomie der Gemeinden vor Ort.

Die religionssoziologische Beobachtung zeigt nun allerdings, dass beide Männer aus einer vorgeblich einheitlichen "Theologie“ der Pfingstbewegung ganz unterschiedliche religiöse Überzeugungen gewinnen sowie soziale und politische Strategien entwickeln. reißig Jahre nach dem ersten Projekt ist die starke Polarisierung der Kriegsgesellschaften einer größeren sozialen und politischen Vielfalt gewichen. Die Möglichkeiten der politischen Mitgestaltung haben sich deutlich erweitert. Auch die Pfingstkirchen sind vielfältiger geworden. Die Reichhaltigkeit ihrer religiösen Symbolik und die Freiheit der Kombination lassen in verschiedenen gesellschaftlichen Klassen, Alterskohorten und sozialen Randgruppen sehr unterschiedliche Ausprägungen von Glaubenspraxis entstehen.

So hat etwa die Gewalt ihr Gesicht verändert. Sie ist nicht mehr militärisch, dafür durchtränkt Kriminalität das Leben der Zivilgesellschaft. Der Glaube an den Schutz des Heiligen Geistes verhilft zu einem Gefühl der Sicherheit. In vielen Armenvierteln der großen Städte zum Beispiel gilt nur eines von zwei Gesetzen: das waffenbewehrte der Drogendealer "auf der Straße“ oder das des "Gottes der Heerscharen“ in den Gemeinden. Gerade für junge Leute aus diesen Stadtvierteln bieten die pfingstkirchlichen Gemeinden oft die einzige Möglichkeit, sich dem Banden-Druck zu entziehen, die "Dämonen der Gewalt" zu bannen und sich anderweitig gesellschaftlich zu integrieren.

Auch der Kampf um soziale Teilhabe hat sich verändert. Will man an den Hoffnungen der neu entstehenden Mittelschichten partizipieren, so ist der Preis dafür ein extremer Druck, ausgelöst durch die Drohung erneuten Abstiegs bei der kleinsten Disziplinlosigkeit. Doch man kann in den Gottesdiensten pfingstlicher Mittelschichtskirchen mit der „Kraft des Heiligen Geistes“ ein Antidot (Gegengift) gegen Stress und Burnout sowie ein spezielles Karriere-Coaching finden. Mega-Kirchen bedienen mit

Links: Bei einer Massentaufe in São Paolo. Unten: Ländliche Armut in Guatemala.

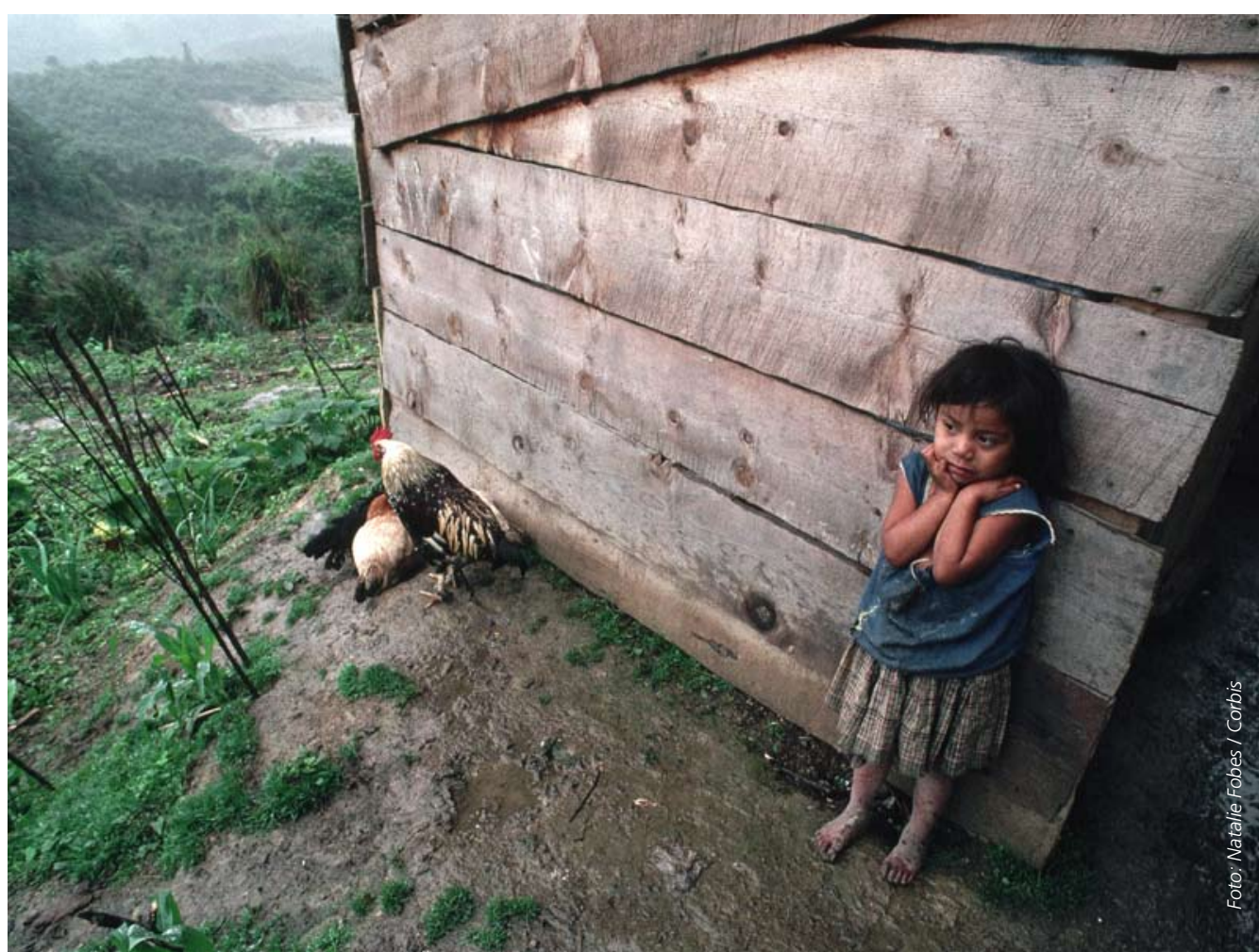


nutzbar zu machen. Sie geht von einfachsten Voraussetzungen aus: Wir nehmen lediglich an, dass alle Menschen relevante Erfahrungen negativer und positiver Art sammeln und diese auf eine für sie relevante Weise deuten, religiös oder auch nicht religiös. Hierauf aufbauend lassen sich die für die Akteure relevanten Erfahrungen und Deutungen methodisch in einem System zusammenfassen. So offenbart die Analyse schließlich ein Netzwerk: eine kognitive Landkarte der religiösen Akteure, die im Zusammenhang ihrer Lebensbedingungen interpretiert werden kann.

Unsere Eingangsbeispiele wurden durch diese Analyse überhaupt erst sichtbar. Zudem wird plausibel, warum der "Agent Gottes“ mit der Macht des "Heiligen Geistes“ nicht nur die Dämonen des kriegsbedingten Stresses (Alkoholismus, Bulimie oder Paranoia usw.) austreibt, sondern auch die Dämonen der

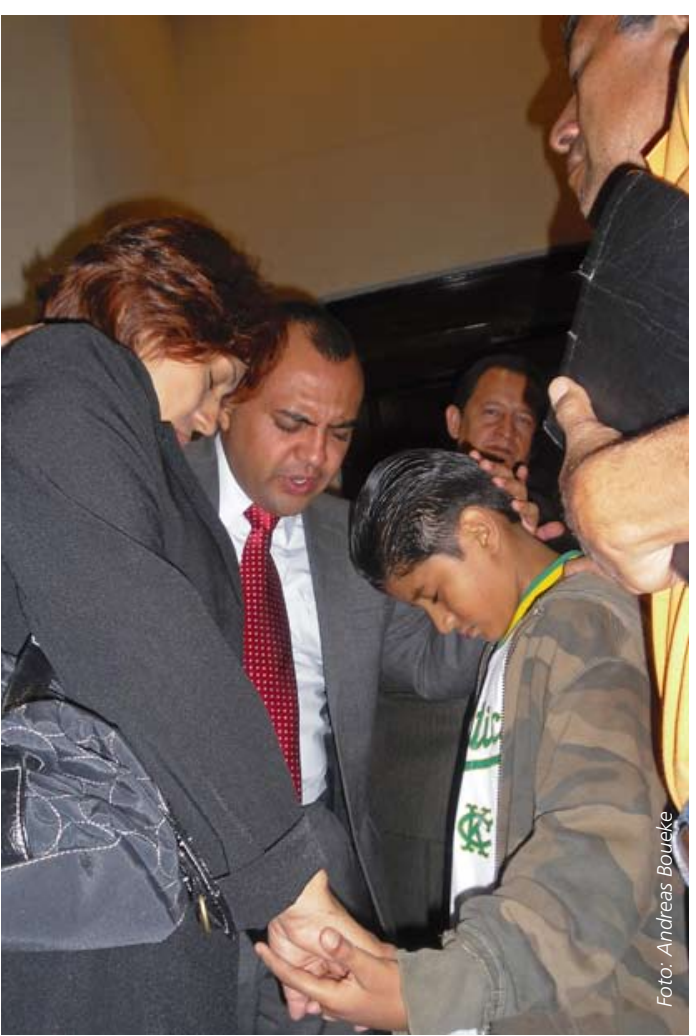

Gewerkschaften und der Guerilla - und warum er die Eliminierung indianischer Bevölkerung durch die Militärregierung unterstützt. Ebenfalls wird deutlich, warum der Bauer sich in seine Kirchengemeinde zurückzieht und auf das Ende der Welt hofft. Und schließlich wird klar, warum der pfingstliche Bauer mit dem vermeintlich genauso pfingstlichen Prediger nichts gemein hat und haben will, sondern sich eher vor ihm fürchtet.

Dieser Forschungsansatz wurde vom Leiter des aktuellen Forschungsteams in etwa 30 Jahren Religionsforschung fortentwickelt. Während einer neunjährigen wissenschaftlichen Tätigkeit in Lateinamerika konnte er unter anderem in empirischen Studien mit Angehörigen der Pfingstbewegung vielfach validiert werden. Seit 2006 wurde er an der Universität Bielefeld in Projekten zu religiösen Friedenstiftern in Bosnien und Herzegowina (Förderung der DFG), zu religiösen Stilen in Argentinien und religiöser Diversität in Mexiko nebst einer vergleichenden Studie zum muslimischen und US-amerikanischen Fundamentalismus wiederum getestet und fortgeschrieben. Derzeit wird auf dieser Grundlage ein Forschungsprojekt in Guatemala und Nicaragua - den Feldforschungsgebieten des Projektleiters in den 1980er-Jahren - durchgeführt.

Der systematische Ländervergleich soll später um einen zeitlichen (Verlaufs-) Vergleich zwischen den 1980er-Jahren und heute erweitert werden. In diesen 30 Jahren wurden die Guerillakriege beendet. Es wurden Friedensprozesse unter Beteiligung religiöser Akteure eingeleitet. Die soziale Ungleichheit verschärfte sich, und die Gewalt wandelte sich von einem militärischen zu einem zivilgesellschaftlichen Phänomen. Angesichts der veränderten Lebensbedingungen ist ein signifikanter Wandel in den religiösen Überzeugungen und der religiösen Praxis insgesamt zu erwarten.

Sehr vieles deutet auf eine sich verstärkende politische Aktivität verschiedener religiöser Gruppierungen hin - also gerade nicht auf das, was (vor allem) in Europa unter dem Stichwort der "Säkularisierung“ diskutiert wird. Wie sehr die Aktivitäten zu Frieden oder zu Konflikt beitragen und wie ein stärker werdender religiöser Einfluss in Gesellschaft und Politik einzuschätzen ist - das bleibt zu untersuchen.
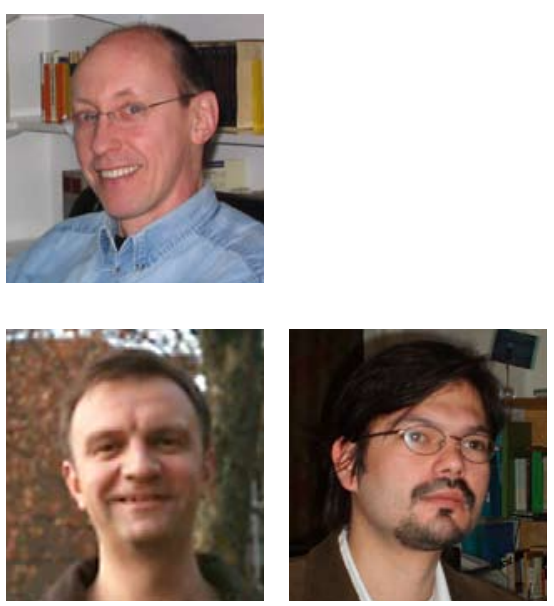

Prof. Dr. theol. Dr. phil. Heinrich Wilhelm Schäfer

lehrt Religionssoziologie und Theologie an der Universität Bielefeld.

Tobias Reu und Adrián Tovar Simoncic sind Wissenschaftliche Mitarbeiter am Center for the Interdisciplinary Research on Religion and Society (CIRRuS).

Adresse: Universität Bielefeld, Fakultät für Geschichte, Philosophie und Theologie / Fakultät für Soziologie, Universitätsstr., 33501 Bielefeld

DFG-Unterstützung in verschiedenen Projekten und Förderverfahren. Den vorliegenden Beitrag schrieb der Verfasser als Fellow am LichtenbergKolleg der Georg-August-Universität Göttingen. 